\title{
Relative orientation of orbits in triple stars ${ }^{\star}$
}

\author{
M. F. Sterzik ${ }^{1}$ and A. A. Tokovinin ${ }^{2}$ \\ 1 European Southern Observatory, Casilla 19001, Santiago 19, Chile \\ 2 Cerro Tololo Inter-American Observatory, Casilla 603, La Serena, Chile
}

Received 6 December 2001 / Accepted 15 January 2002

\begin{abstract}
Statistical analysis of the relative alignment of inner and outer orbits in triple systems resulting from a dynamical decay of small- $N$ clusters $(N \leq 10)$ is presented and compared to the statistics of real multiple stars. The distribution of the relative angle $\Phi$ between the angular momentum vectors of inner and outer orbits in triple stars formed by decay is shown to depend on the initial cluster conditions like geometry, mass function, rotational and thermal energy. For a realistic set of initial conditions, a modest alignment of orbital momentum vectors is found, in good agreement with the latest observational data on visual multiple stars exhibiting an average $\langle\Phi\rangle$ between $67^{\circ}$ and $79^{\circ}$. The relation between eccentricities of outer orbits and period ratios for both simulated and real triples is consistent with a slightly adjusted formulation of the stability criterium by Mardling \& Aarseth (2001). Dynamical decay can therefore explain the weak correlation of orbital orientations observed in multiple stars. Using modern high-resolution techniques, the observed statistics of $\Phi$ should be extended as it will allow one to sensitively constrain properties of initial clusters.
\end{abstract}

Key words. stars: binaries: general - visual - stars: formation - stellar dynamics

\section{Introduction}

Most stars form in binary and multiple systems. The mechanisms that define binary fraction, period and mass ratio distributions remain to be identified and modeled. As pointed out by Batten (1973), studies of multiple (i.e. triple, quadruple, etc.) stellar systems might provide important clues to the formation processes. Here we concentrate on the statistics of the relative angle $\Phi$ between angular momentum vectors of inner and outer orbits in hierarchical triple (or higher order) systems. Like other orbital parameters, this angle is a fossil record of the conditions at the epoch of formation: although in triple systems $\Phi$ does change periodically owing to Kozai cycles (see below), its statistics must remain secularly stable.

Planetary orbits in the Solar system are close to coplanar and stable. If multiple stars form by hierarchical fragmentation of a rotating cloud, as suggested by Bodenheimer (1978), or by fragmentation of a circumbinary disk (Bonnel \& Bate 1994), similar coplanar configurations must result. On the other hand, dynamical decay of small non-hierarchical stellar clusters was believed

Send offprint requests to: M. Sterzik,

e-mail: msterzik@eso.org

* Table 4 is only available in electronic form at the CDS via anonymous ftp to

cdsarc.u-strasbg.fr (130.79.128.5) or via

http://cdsweb.u-strasbg.fr/cgi-bin/qcat?J/A+A/384/1030 to lead to uncorrelated orbital momenta in the resulting multiples. Fekel (1981) determined that at least $1 / 3$ of triple stars indeed have non-coplanar orbits. Hence, the knowledge of the angle $\Phi$ can constrain binary formation theories. It is shown below that $N$-body decay in fact does produce multiples with partially correlated orbits and that the $\Phi$ statistics is related to the properties of initial clusters.

Fragmentation during the collapse of molecular cloud cores appears to be most likely the first step in star formation. Subsequent formation and evolution of binaries and multiples involves a combination of different physical processes which cannot yet be modelled completely. Of all those processes, dynamical interactions between fragments can be studied by $N$-body calculations and can lead to quantitative predictions of the properties of binaries and multiples. Sterzik \& Durisen (1995) and Sterzik \& Durisen (1998) explored in detail the consequences of the dynamical decay of initially nonhierarchical few-body systems (also called progenitor clusters in the following), mimicking initial configurations at the end of cloud fragmentation. They used precise, direct numerical orbit integrators, and simulated many individual few-body systems until stable, noninteracting, subsystems or remnants consisting of (escaped) single stars, binaries and hierarchical higher order systems were reached. Detailed statistical analysis of these remnants allows to predict binary properties like their 
frequencies, mass-ratios and dependence on primary mass. They tend to agree quite well with observations, provided reasonable assumptions about the initial mass function are made, as demonstrated e.g. in Durisien et al. (2001). However, orbital periods of binaries produced by dynamical decay cannot be as short as in some real binaries (Kroupa \& Burkert 2001), indicating the importance of other processes, like dissipative orbital shrinkage.

According to Sterzik \& Durisen (1998), between $10 \%$ and $20 \%$ percent of all decaying few-body systems end in meta- or long-term stable hierarchical systems. It is the goal of this contribution to statistically analyze the relative orientation of orbital angular momenta in triple stars resulting from $N$-body decay calculations and to explore their dependencies on initial conditions such as $N$ (number of stars), mass function, virial state and geometry.

Observationally, the determination of the relative orientation of orbital momenta is extremely difficult. In principle, complete orbit solutions have to be determined for both inner and outer orbits (or, more generally, for two orbits of adjacent hierarchy levels). These cases are rare. On the one hand, outer orbital periods are often too long compared to the 200-year time span of double star observations. On the other hand, inner orbits are frequently too close to be resolvable with current techniques, awaiting long-baseline interferometry and precise astrometry.

A first attempt to study the distribution of the angle $\Phi$ in three samples of real multiple stars was done by Tokovinin (1993). Two of those samples lacked complete two-orbit solutions and were analyzed by indirect methods. A robust result of this study was that neither coplanarity nor complete random orientation prevailed. Instead, the available data could only be interpreted by involving a modest degree of orbital momenta alignment. Here we enhance such an analysis using updated information from the current version of the Multiple Star Catalog (MSC) by Tokovinin (1997) and compare the results with the predictions of $N$-body simulations. Thus, initial configurations of the $N$-body systems can be constraint. Also the influence of dynamical stability on the orbit orientation will be discussed.

\section{Dynamical decay simulations}

A detailed description of the methodology employed in our dynamical decay calculations of non-hierarchical fewbody systems can be found in Sterzik \& Durisen (1995) and in Sterzik \& Durisen (1998). In short, the regularization scheme CHAIN of Mikkola \& Aarseth (1993) is used to integrate orbits for typical 300 crossing times. After this time, most clusters have decayed into meta- or long-term stable subsystems, classified as singles, binaries and higher-order hierarchical bound subsystems. A cluster model is characterized by certain initial conditions, and it was demonstrated that $N$ (the number of point-mass stars) and the stellar mass spectrum had major effects on remnants, whereas the outcome is less sensitive to initial cluster geometries and virial status, provided they are not extreme. Model parameters include the shape of initial configuration (axis ratio for oblate and prolate spheroids), rotation (the ratio of rotational to gravitational energy $\beta$ ) and virial status (the ratio of random kinetical to gravitational energy $\alpha$ ) as in Sterzik \& Durisen (1995). For each model, 1000 realizations are typically generated and integrated individually to allow statistical analysis.

For the current study we will concentrate on the sample of triple systems that are generated in this way. We focus on the angle between the inner and outer orbital angular momentum vectors $\Phi$, which is unambiguously defined for these systems. Although systems higher than triple can be found among remnants, their number is small compared to triples and we restrict our analysis to triples only.

\section{1. $N=4$, equal masses}

In order to explore the main influences on the $\Phi$ distribution, we first consider equal masses cases with some extreme choices of initial conditions. Using $N=4$, we avoid the degenerate case $N=3$ where the orbital momenta will be strictly counter-aligned if the initial system had zero total angular momentum.

In Fig. 1 we compare the cumulative distribution function of $\Phi$ for a representative set of different initial system conditions. The thin line indicates the distribution for completely uncorrelated, i.e. random orientations:

$F(\Phi)=0.5(1-\cos \Phi)$

All relative orientations are equally probable, and the mean angle is therefore $\langle\Phi\rangle=90^{\circ}$. For perfectly aligned orbital momenta, $\langle\Phi\rangle=0$; thus, values of $\langle\Phi\rangle$ decreasing from $90^{\circ}$ to $0^{\circ}$ correspond to progressively increasing degree of orbit alignment.

Triple systems produced by a dynamical decay of a spherical cluster without initial motions have - within statistical fluctuations - a random distribution of $\Phi$ (thick line, labelled spherical). But when an initial four-body cluster has strong rotation, the correlation of orbital momenta increases considerably (label spherical, $\beta=0.5$ ). Similarly, a prolate ellipsoidal cluster with axis ratio of 100:1 leads to an increased correlation, although a certain number of counter-aligned orbits is also produced (label prolate). In general, flattened geometries alone do not produce correlations of momentum vectors, but a strong correlation appears when initial rotation is added, as evidenced in Fig. 1 by the curves with the corresponding labels oblate, $\beta=0.3$ and oblate, $\beta=0.5$. Again we note that these configurations yield a considerable fraction of counter-aligned orbits, which is not easily understood intuitively when a strong initial systemic rotation is present.

We give the mean angles $\langle\Phi\rangle$ obtained in our simulations for different model configurations in Table 1, sorted by increasing correlation. The statistical error of the mean angle is typically $3^{\circ}$ for the sample sizes involved. We have also modeled the effect of initial random motions $(\alpha=0.5)$ 


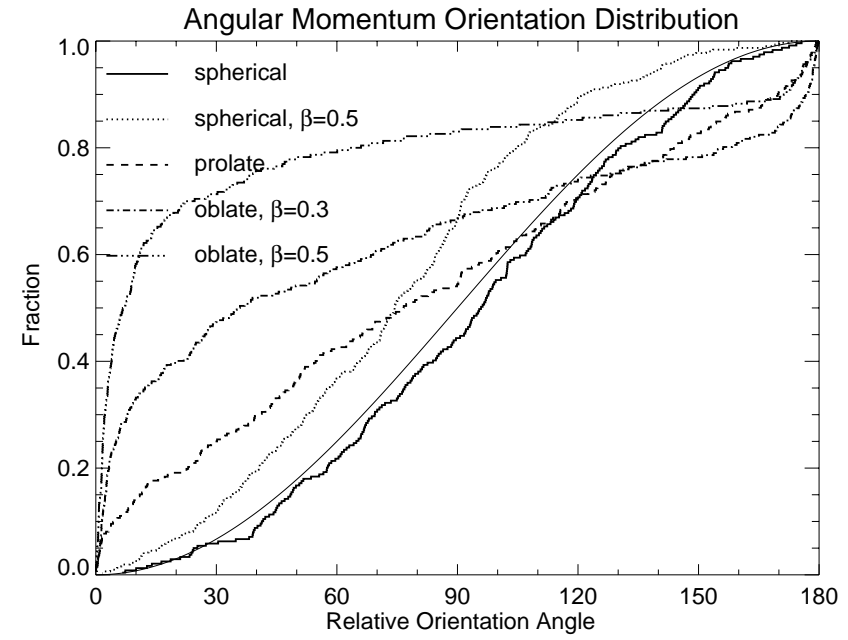

Fig. 1. Cumulative distributions of the angle $\Phi$ between the angular momentum vectors in simulated triple systems. All models refer to $N=4$, equal masses initial conditions. The thin line indicates the distribution for random mutual orientation of orbits.

which tends to wipe out imposed correlations, as well as more modest choices of the oblateness parameter and $\beta$. They have only marginal effects on $\langle\Phi\rangle$.

Table 1. Mean angle $\langle\Phi\rangle$ for different model configurations in the case $N=4$, equal masses. We also give the total sample size of triple systems $N_{\text {tot }}$ considered per model.

\begin{tabular}{lllcc}
\hline geometry & $\alpha$ & $\beta$ & $N_{\text {tot }}$ & $\langle\Phi\rangle$ \\
\hline \hline spherical & 0 & 0 & 239 & 95 \\
oblate 100:1 & 0 & 0 & 274 & 95 \\
spherical & 0.5 & 0 & 334 & 92 \\
oblate 100:1 & 0 & 0.1 & 228 & 88 \\
oblate 10:1 & 0 & 0.1 & 271 & 85 \\
prolate 100:1 & 0 & 0 & 272 & 82 \\
spherical & 0 & 0.5 & 310 & 75 \\
oblate 100:1 & 0 & 0.3 & 262 & 67 \\
oblate 100:1 & 0 & 0.5 & 230 & 37 \\
\hline
\end{tabular}

\subsection{Realistic mass spectra}

More realistic assumptions about the initial system configuration require us to relax the condition of a fixed initial number of stars per simulation $N$ and to apply a stellar mass spectrum. The essential effects of different mass functions on the dynamical decay and evolution of few-body systems have been demonstrated in Sterzik \& Durisen (1998).

Early dynamical decay studies predicted a very strong dependence of the binary fraction on primary masses, inconsistent with observations. The problem was circumvented by introducing a "two-step" initial mass function (IMF) that results from a combination of two mass spectra: a cloud mass spectrum (CMS) which defines the total mass of each cluster and a stellar mass spectrum (SMS). This additional constraint on the cluster masses results in a more uniform distribution of stellar masses within each cluster, while still matching the overall stellar mass function. As shown by Durisen et al. (2001), this approach can explain the trends in observed binary fraction and mass-ratio distributions with primary mass. The existence of multiple systems composed of only low-mass stars, like the quintet associated with M-dwarf Gliese 644 (Mazeh et al. 2001), is a strong argument in favor of the "two-step" IMF process.

The following simulations use the same powerlaw CMS and log-normal SMS as in Durisen et al. (2001), but the lower mass cutoff extends to $0.01 M_{\odot}$ to include brown dwarf masses. For each cluster, stars with masses randomly selected from SMS are added until the desired total mass (selected from CMS) is reached within some tolerance interval. The number of stars $N$ in each cluster is therefore variable. However, we constrain this number between $3 \leq N \leq 10$ (for $N=1,2$ the dynamical decay simulations are meaningless). We have also calculated the $\Phi$ statistics for simpler models, such as fixed $N$ with various mass spectra. But our main conclusions with respect to $\langle\Phi\rangle$ do not change, and therefore we concentrate below on the results from the two-step, variable $N$ simulations which we consider as the most advanced and realistic approach for dynamical decay models. Table 2 lists (in order of increasing orbit alignment) the $\langle\Phi\rangle$ for all models based on the variable $N$, two-step IMF simulations. The statistics of the numbers involved in the analysis implies mean errors of $3-5$ degrees for $\langle\Phi\rangle$.

First of all, we note that all "cold" clusters, i.e. models without initial motions $(\alpha=\beta=0)$, exhibit slightly anticorrelated orbital momenta independent of their initial geometry. One reason is that about $10 \%$ of all triple systems originate from $N=3$ clusters; these triples have strictly antiparallel momentum vectors because of angular momentum conservation. But even without these degenerate cases the mean angle remains significantly above $90^{\circ}$. This behavior can be seen in Fig. 2, where we plot the cumulative distribution of $\Phi$ for selected cases.

With a small amount of initial random motions $(\alpha=$ 0.1 ), the angles become random for all cluster geometries. In contrast to the equal masses case, significant orbit alignment is readily achieved by adding small amounts of initial rotation. It follows from Table 2 and Fig. 2 that the spherical, $\beta=0.1$ model yields similar orbit correlation as, e.g. oblate 10:1, $\beta=0.05$. Additional random initial motions together with initial rotation do not destroy the orbit alignment, as long as $\alpha \leq \beta$. Finally, small cluster oblatenesses $(10: 1)$ together with small initial rotation $(\beta=0.1 \ldots 0.2)$ produce high degrees of correlation, only found with higher $\beta$ values in the equal masses case. The high sensitivity of $\langle\Phi\rangle$ to the mass spectrum as compared to the equal masses cases is evident when comparing the same oblate 100:1, $\beta=0.1$ model in Tables 1 and 2 . Whereas in the equal masses case the correlation was almost absent, $\langle\Phi\rangle=88^{\circ}$, the two-step mass spectrum, variable $N$ case yields $\langle\Phi\rangle=50^{\circ}$. 


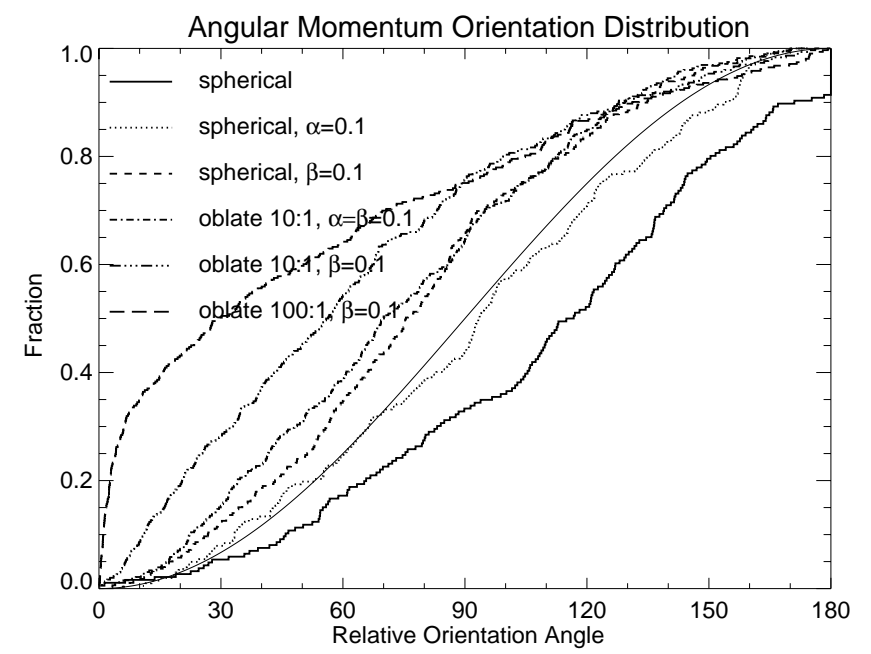

Fig. 2. Cumulative distribution of the angle $\Phi$ between the angular momentum vectors in simulated triple systems. All models refer to the variable $N$, two-step IMF explained in the text.

The total angular momenta of the progenitor clusters and the triple systems formed by dynamical decay correlate in direction and magnitude. Obviously, for progenitor clusters with $N=3$ this correlation is $100 \%$, but also for $N>3$ this correlation is strong. E.g., if we exclude the $N=3$ progenitor clusters in the case of the oblate $10: 1, \beta=0.1$ model, the mean angle between the two angular momenta is still $32^{\circ} \pm 36^{\circ}$, having a mean ratio of $0.61 \pm 0.51$. On average, triple systems inherit the direction and amplitude of their angular momentum from the progenitor cluster.

We conclude from our analysis that the relative orientation of orbital momenta in triple systems resulting from a dynamical decay with realistic initial conditions like those involving a two-step IMF, variable $N$ clusters advocated in Durisen et al. (2001), depends sensitively on the geometry and rotational status of the parental cluster from which they form. In other words, the $\Phi$ statistics constitutes a powerful probe of progenitor cluster properties that are difficult or impossible to determine by other means.

\section{Comparison with real multiple stars}

We enhance the study of Tokovinin (1993) by taking advantage of the increased volume of data collected in the MSC database. The catalog is continuously updated from the current literature. Specifically, we re-actualized all information on visual orbits using the recent 6 -th visual orbit catalog of Hartkopf \& Mason (2001). While employing the same method as in 1993, we concentrate only on visual multiple stars because their long orbital periods could directly result from a cluster decay process. Some of the triples considered below actually belong to higher-order systems, containing either more distant companions, or close spectroscopic sub-systems, or both. For example, the quadruple system $\zeta$ Cnc $(08065+1757)$ contributes two "triples" because the visual orbits of the outer and two
Table 2. Mean angles $\langle\Phi\rangle$ for different model with a two-step IMF and variable cluster size $N$. We also give the total number of triple systems $N_{\text {tot }}$ per model. Indices "s" and "u" refer to sub-samples considered as dynamically long-term stable or unstable.

\begin{tabular}{lllrrrrr}
\hline geometry & $\alpha$ & $\beta$ & $N_{\text {tot }}$ & $\langle\Phi\rangle$ & $N_{\mathrm{s}}$ & $\langle\Phi\rangle_{\mathrm{s}}$ & $\langle\Phi\rangle_{\mathrm{u}}$ \\
\hline \hline obl. 10:1 & 0 & 0 & 185 & 116 & 105 & 102 & 134 \\
obl. 100:1 & 0 & 0 & 156 & 113 & 91 & 105 & 123 \\
sph. & 0 & 0 & 186 & 111 & 114 & 102 & 126 \\
obl. 10:1 & 0.1 & 0 & 236 & 95 & 138 & 94 & 95 \\
sph. & 0.1 & 0 & 202 & 93 & 117 & 93 & 93 \\
obl. 100:1 & 0.1 & 0 & 246 & 90 & 136 & 86 & 95 \\
sph. & 0 & 0.1 & 279 & 78 & 163 & 72 & 86 \\
obl. 10:1 & 0.05 & 0.05 & 267 & 78 & 160 & 74 & 84 \\
obl. 10:1 & 0.1 & 0.1 & 326 & 75 & 213 & 77 & 70 \\
obl. 10:1 & 0.2 & 0.2 & 303 & 74 & 174 & 76 & 71 \\
obl. 10:1 & 0 & 0.05 & 261 & 74 & 171 & 70 & 81 \\
obl. 10:1 & 0 & 0.1 & 300 & 63 & 188 & 57 & 72 \\
obl. 100:1 & 0 & 0.1 & 313 & 50 & 206 & 47 & 58 \\
obl. 10:1 & 0 & 0.2 & 376 & 49 & 246 & 52 & 42 \\
\hline
\end{tabular}

inner systems are known. It should be remembered that the data are subject to very serious observational selection regarding both discovery of multiple systems and the availability of sufficient information like orbits.

\subsection{Multiples with two visual orbits}

First, we consider the systems where two visual or astrometric orbits at adjacent hierarchy level are known. The sample size increased from 14 (1993) to 22. Table 3 lists these systems. In view of the small number of available systems, 8 objects with questionable orbits are included in this sample with critical remarks.

The relative angle $\Phi$ between angular momentum vectors is computed according to

$\cos \Phi=\cos i_{1} \cos i_{2}+\sin i_{1} \sin i_{2} \cos \left(\Omega_{1}-\Omega_{2}\right)$.

Here $i_{1}$ and $i_{2}$ are the orbital inclinations and $\Omega_{1}$ and $\Omega_{2}$ are the position angles of the lines of nodes. Those angles are only known with $180^{\circ}$ ambiguity, unless true ascending node is identified by radial velocities. Only for three systems in our list both angles are thus identified and the ambiguity is resolved (cf. Tokovinin, 1993). For the remaining cases, the sign of $\cos \left(\Omega_{1}-\Omega_{2}\right)$ is ambiguous and we do not know which of the two resulting angles $\Phi$ is the correct one. A "false" angle is obtained if one of the angular moment vectors is reflected symmetrically with respect to the viewing direction. In the following, we compute both values of $\Phi$ for all systems and construct the cumulative distribution as a mixture of correct and false angles, as shown in Fig. 3. In this way, the mean angle between orbital momenta is $\left\langle\Phi_{\mathrm{amb}}\right\rangle=79^{\circ} \pm 6^{\circ}$. The error was estimated assuming that $\Phi$ is distributed approximately like Eq. (1) with a dispersion of $\sqrt{\pi^{2} / 4-2}=39^{\circ}$, hence the estimate of the mean from a sample of 44 angles has a rms error $\sqrt{43}$ times smaller. Compared to the 
Table 3. All systems with two visual orbits known from MSC. We give IDS(1900) identifier, spectral type of the primary, and the orbital elements (logarithm of the period $P$ in days, position angle of ascending node $\Omega$, inclination $i$ and eccentricity $e$ ) for the outer and the inner orbits.

\begin{tabular}{|c|c|c|c|c|c|c|c|c|c|c|}
\hline IDS(1900) & $\mathrm{Sp}(\mathrm{A})$ & $\log P_{\text {out }}$ & $e_{\text {out }}$ & $\Omega_{\text {out }}$ & $i_{\text {out }}$ & $\log P_{\text {in }}$ & $e_{\text {in }}$ & $\Omega_{\text {in }}$ & $i_{\text {in }}$ & Remark \\
\hline $00263+6642$ & $\overline{~ d M 2.5}$ & 5.07 & 0.000 & 168.0 & 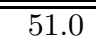 & 3.75 & 0.000 & 24.0 & 27.0 & \\
\hline $00508+5949$ & A1V & 4.48 & 0.241 & 175.0 & 54.9 & 3.25 & 0.230 & 185.0 & 55.0 & Inner astrom. orbit weak \\
\hline $01304-3026$ & $\mathrm{~K} 3 \mathrm{~V}$ & 4.61 & 0.210 & 141.8 & 29.3 & 3.22 & 0.301 & 57.4 & 21.8 & \\
\hline $01562+3614$ & G9V & 5.08 & 0.330 & 159.0 & 140.0 & 3.67 & 0.404 & 191.4 & 67.0 & \\
\hline $02208+6657$ & A $5 p S r$ & 5.35 & 0.750 & 0.8 & 115.0 & 4.28 & 0.300 & 175.0 & 106.0 & Outer orbit uncertain \\
\hline $04320+5316$ & $\mathrm{~A} 8 \mathrm{~V}$ & 5.20 & 0.320 & 112.5 & 133.0 & 3.99 & 0.860 & 20.8 & 141.0 & \\
\hline $05566-3103$ & $\mathrm{~K} 5 \mathrm{~V}$ & 5.15 & 0.270 & 142.8 & 110.2 & 4.39 & 0.450 & 125.0 & 103.0 & \\
\hline $07142+2210$ & F2IV & 5.64 & 0.110 & 18.4 & 63.3 & 3.35 & 0.353 & 70.0 & 92.4 & Inner orbit controversial \\
\hline $08065+1757$ & F7V & 5.61 & 0.240 & 74.2 & 146.0 & 4.34 & 0.320 & 13.0 & 167.0 & \\
\hline $08065+1757$ & F7V & 5.61 & 0.240 & 74.2 & 146.0 & 3.80 & 0.080 & 77.0 & 142.0 & \\
\hline $08415+0647$ & G5III & 5.56 & 0.300 & 229.3 & 39.0 & 3.74 & 0.665 & 284.8 & 50.0 & Quintuple \\
\hline $08524+4826$ & A7IV & 5.47 & 0.790 & 4.8 & 57.8 & 4.16 & 0.320 & 21.0 & 108.0 & Outer orbit uncertain \\
\hline $11128+3205$ & G0V & 4.34 & 0.398 & 101.8 & 122.1 & 2.83 & 0.610 & 318.0 & 91.0 & \\
\hline $14516-2058$ & $\mathrm{~K} 4 \mathrm{~V}$ & 5.89 & 0.200 & 317.3 & 72.5 & 2.49 & 0.765 & 18.0 & 110.0 & Outer orbit uncertain \\
\hline $15140+2712$ & G0V & 4.87 & 0.652 & 64.0 & 58.2 & 4.26 & 0.700 & 135.0 & 90.0 & Inner system controversial \\
\hline $20435+3607$ & $\mathrm{~B} 5 \mathrm{Ve}$ & 5.15 & 0.450 & 138.6 & 133.8 & 3.63 & 0.524 & 150.0 & 135.0 & \\
\hline $22009+6408$ & $\mathrm{~A} 3 \mathrm{Vm}$ & 6.14 & 0.240 & 85.0 & 109.0 & 2.91 & 0.589 & 93.5 & 71.9 & Outer orbit uncertain \\
\hline $22237-0032$ & F3V & 5.44 & 0.500 & 304.6 & 135.9 & 3.97 & 0.590 & 202.7 & 34.3 & \\
\hline $22370+2054$ & G0 & 5.37 & 0.000 & 27.1 & 126.9 & 4.48 & 0.000 & 13.8 & 42.2 & \\
\hline $22573+4147$ & B6III & 4.40 & 0.480 & 191.0 & 103.6 & 3.51 & 0.127 & 15.0 & 81.0 & \\
\hline $23047+7451$ & K0III & 4.79 & 0.610 & 81.0 & 30.0 & 2.75 & 0.300 & 107.9 & 99.0 & \\
\hline $23344+4510$ & $\mathrm{~A} 2$ & 4.74 & 0.575 & 127.5 & 130.3 & 3.74 & 0.600 & 129.7 & 127.4 & Both orbits uncertain \\
\hline
\end{tabular}

14 systems discussed in Tokovinin (1993), the correlation of momenta in the latest data set has slightly decreased. If we exclude the 8 systems with bad or uncertain orbits, it does not lead to any noticeable change in the distribution and mean angle.

For qualitative comparison, we show in Fig. 3 the oblate 10:1, $\beta=0.1$ model discussed in the previous section. We mix the true angles $\Phi$ with false angles, obtained by reflecting the inner orbit momentum vector with respect to a random viewing direction, in order to mimic the ambiguity of real data. The curve labelled ambiguity corr. shows the corresponding cumulative distribution. For this distribution, the mean angle is $\left\langle\Phi_{\mathrm{amb}}\right\rangle=73^{\circ}$. A correlation in relative orbit orientation is diluted by the ambiguity, and the simulated and observed distribution functions match very well for $\Phi \leq 90^{\circ}$. The two curves differ above that angle, as the observed angles appear to be depleted around $\Phi \approx 90^{\circ}$. Triple stars with $\Phi \approx 90^{\circ}$ are subject to the Kozai effect which, coupled with dissipative interactions, leads to significant changes of orbital parameters, cf. Kiseleva et al. (1998). Given the limited statistical significance of the observations, we do not intend to fit the data more accurately.

\subsection{Sign correlation}

An elegant technique to study the relative orbit orientation in visual binaries is counting the apparent directions of revolution. This method was pioneered by Worley (1967) and used in Tokovinin (1993). Let $n_{+}$be the number of triple stars where the apparent directions of

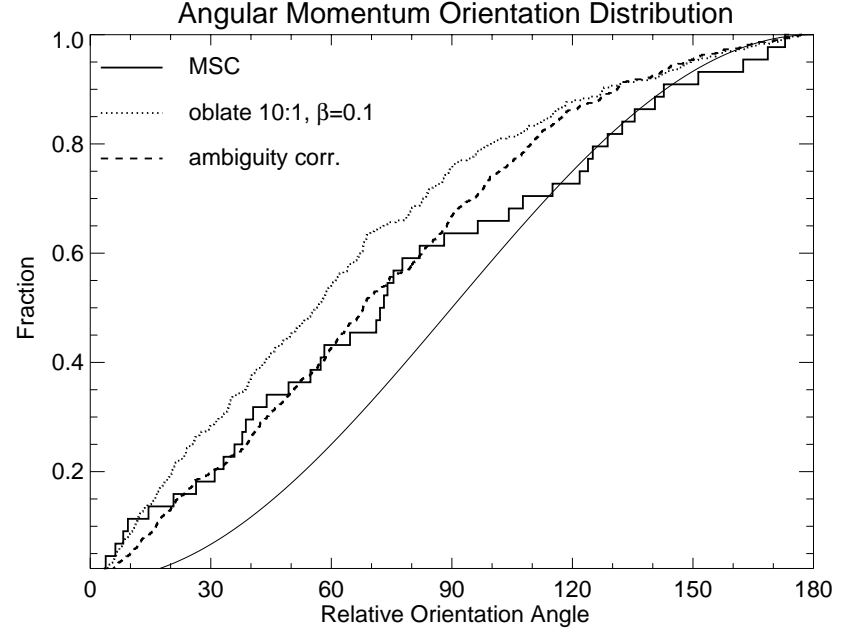

Fig. 3. Cumulative distribution of the angle $\Phi$ between the angular momentum vectors in triple systems having two visual orbits from MSC. The thin line indicates distribution for uncorrelated orbit orientations. The angle distribution for a specific dynamical decay model is overplotted (with and without simulation of the angle ambiguity inherent to real data).

revolution in inner and outer systems are the same (corotating), and $n_{-}-$the number of counter-rotating systems. Then the sign correlation $C$ is defined as

$C=\left(n_{+}-n_{-}\right) /\left(n_{+}+n_{-}\right)$.

Simple arguments show that the rms statistical error of the estimation of $C$ is $\pm \sqrt{(1+C)(1-C) /\left(n_{+}+n_{-}\right)}$.

In Fig. 4 the geometry of the problem is presented. The orbital momentum vectors $\boldsymbol{J}_{\text {out }}$ and $\boldsymbol{J}_{\text {in }}$ and the 


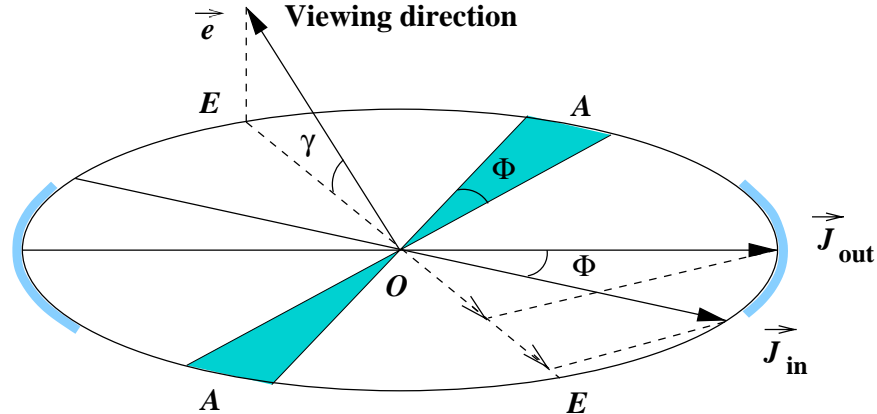

Fig. 4. Geometry of true and apparent sense of rotation.

viewing direction $\boldsymbol{e}$ are drawn as unit vectors starting from the coordinate origin $O$. The apparent sense of rotation (clockwise or counter-clockwise) in each orbit depends on the signs of the projections of $\boldsymbol{J}_{\text {out }}$ and $\boldsymbol{J}_{\text {in }}$ onto the viewing direction or, equally, onto the direction $E E$ lying in the plane of these vectors: when both vectors project in the same direction, as in Fig. 4, the system appears as co-rotating. If the line $E E$ happens to fall in the shaded sector $A A$, the system appears as counter-rotating. Inclination $\gamma$ of the viewing direction $\boldsymbol{e}$ with respect to the plane is of no importance.

For any given angle $\Phi$ between the vectors $\boldsymbol{J}_{\text {out }}$ and $\boldsymbol{J}_{\text {in }}$ and for random orientation of the viewing direction with respect to a triple system, the expected number of corotating systems in a sample of size $M$ is $n_{+}=M(\pi-$ $\Phi) / \pi$, as evident from the simple geometry above. Hence, the sign correlation is

$C=1-2 \Phi / \pi$

This relation is linear in $\Phi$, so a statistical averaging over any distribution of the angle $\Phi$ will lead to the same relation between the average values. Quite remarkably, sign correlation enables a direct measurement of the average angle $\Phi$ :

$\langle\Phi\rangle=\frac{\pi}{2}(1-\langle C\rangle)$

It is not an easy matter to determine the relative sense of rotation in real triples. First, both inner and outer subsystems must be spatially resolved, presenting problems for close sub-systems. Secondly, a slow angular motion in an outer system must be actually detectable. Bearing in mind the limited accuracy of old observations, we impose a condition that minimum observed change in position angle must exceed $2^{\circ}$. For a 150 year observing span this translates to a maximum orbital period of 27000 years. Shorter observing spans and projection effects restrict the periods of outer systems even more.

The detectability of the motion in outer system is better for some viewing directions - those nearly parallel to $\boldsymbol{J}_{\text {out }}$ when the orbit lies almost in the plane of sky. It means that in fact the viewing direction in a real sample is not random with respect to a triple system, as assumed above. The directions aligned with $\boldsymbol{J}_{\text {out }}$ will be over-represented, as shown schematically in Fig. 4 by grey arcs. This bias increases the observed sign correlation with respect to (4). Unfortunately, the magnitude of the bias depends on the distributions of $\Phi$ and viewing angle, both unknown. Taking an ad hoc $1+\cos (2 \Psi)$ law for the distribution of the angle $\Psi$ between $\boldsymbol{J}_{\text {out }}$ and the projection $E E$ in Fig. 4, we estimated the sign correlation for a realistic distribution of $\Phi$ that resembles the dotted line in Fig. 3 and obtained $C=0.42$, whereas the unbiased value would be $C=0.30\left(\langle\Phi\rangle=52^{\circ}\right.$ from biased $C$, whereas true $\left.\langle\Phi\rangle=63^{\circ}\right)$. It is likely that the real bias will be somewhat less than these crude estimates.

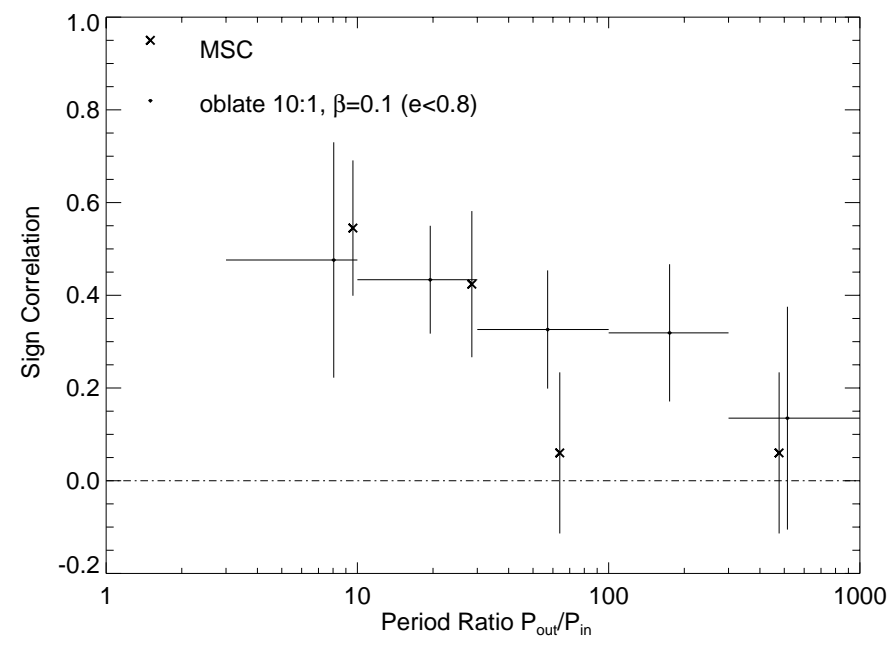

Fig. 5. Sign correlation as a function of period ratio for real systems (crosses) and simulated triples stars.

A total of 135 visual triples were selected from MSC, some of them belonging to higher-order systems. The data on these systems are given in Table 4, available only electronically at the CDS. Surprisingly, many potentially useful systems were rejected for the lack of observations of inner pairs - a deficiency that can be easily corrected with speckle interferometry. The 22 "visual-visual" systems discussed above are obviously included in this sample.

For the whole sample, $C=0.26 \pm 0.10$, which corresponds to $\langle\phi\rangle=66.7^{\circ} \pm 8.7^{\circ}$ (without any correction of the mentioned bias). It means that the angular momenta are indeed partially correlated. For the 22 "visual-visual" binaries the sign correlation is smaller and poorly determined: $C=0.18 \pm 0.21$.

Next, we study the dependence of the sign correlation on the orbital periods of inner and outer systems, $P_{\text {in }}$ and $P_{\text {out }}$. The periods for systems without orbits are estimated from their separations, hence they are correct only in a statistical sense. Some trends with $P_{\text {in }}$ and $P_{\text {out }}$ are found, but they are below significance limits. On the other hand, the dependence of sign correlation on the period ratio seems more marked. In Fig. 5 we plot the sign correlation for the four sub-samples of 33 systems each, selected in order of increasing period ratio. In weakly-hierarchical triples, the sign correlation reaches 0.5 , corresponding to 
$\langle\Phi\rangle=45^{\circ}$. On the other hand, visual triples with large period ratio have almost uncorrelated orbits.

In the same Fig. 5 we overplot the results of the oblate, 10:1, $\beta=0.1$ model, where we have calculated the mean sign correlation according to Eq. (4) for individual period ratio bins. We have only selected triple systems having outer eccentricities $e<0.8$, in order to avoid the high eccentricity systems that are absent in reality (see below). Within the statistical significance, we recover a similar trend of decreasing sign correlation with increasing period ratios.

\section{Effects of dynamical stability}

In Fig. 6 we plot the eccentricities of outer systems versus the ratio of outer and inner periods. Each point corresponds to a triple system formed in a dynamical decay calculation with initial cluster models explained in Sect. 2.2. No significant differences in these parameters are apparent between the 14 models with respect to geometry and/or virial status; therefore we plot all simulated data together. Simulated triples are compared to the 22 real systems from MSC with both visual orbits known, plotted by different symbols. They populate, roughly, the same area as the simulated systems. Both populations tend to avoid small period ratios below approximately 3 for all eccentricities. One characteristic difference, however, is the apparent over-abundance of simulated high eccentricity systems with respect to the MSC. It is well known (cf. Anosova 1989) that the eccentricity distribution in systems produced by dynamical processes follows a $f(e)=2 e$ law. This is not the case for outer sub-systems in real multiples, as noted by Shatsky (2001) and as apparent in Fig. 6.

Simulated triples apparently concentrate at $e=1$. Those high-eccentricity systems are not expected to be long-term stable. Although formulations to explain stability in the general three body problem from first principles are being developed (Mardling 2001), only semiempirical formulas exist in closed form. The full line in Fig. 6 indicates the stability boundary given by Mardling \& Aarseth (2001) in their attempt to describe the survival rate in hierarchical triples having coplanar, prograde orbits within clusters. We rewrite their formula (90) as

$$
\left(P_{\text {out }} / P_{\text {in }}\right)_{\text {crit }}^{2 / 3}=2.8\left(1+q_{\text {out }}\right)^{1 / 15}\left(1+e_{\text {out }}\right)^{0.4}\left(1-e_{\text {out }}\right)^{-s},(6)
$$

where $s=1.2$ and $q_{\text {out }}$ is the mass ratio of the outer body to the sum of the inner ones. The dependence on this parameter is weak, so we use $\left\langle q_{\text {out }}\right\rangle=0.26$ obtained from our simulations. For smaller period ratios (i.e. longer inner periods $\left.P_{\text {in }}\right)$ only triples with moderate $e_{\text {out }}$ should survive, - trend that is known observationally, see Shatsky (2001) and Tokovinin \& Smekhov (2002), and is reproduced by the orbits obtained from our dynamical simulations. We note that the empirical stability boundary might be better explained using $s=0.9$ in the formula above (dashed line), consistent with inclined systems being more stable than coplanar prograde ones.

Systems with period ratios smaller than the critical ratio (i.e. to the left of the curve in Fig. 6) should not be long-term stable according to the criteria above, but we find 6 out of 22 systems in Table 3 in this area. Undoubtedly, in some cases the apparent instability results from poorly known orbital elements. For example, the leftmost point in Fig. 6 belongs to IDS 15140+2712, where the astrometric orbit of the inner system (and, in fact, its existence) are controversial.

Similarly, $39 \%$ (or $24 \%$ using $s=0.9$ ) of the simulated triples fall outside the stability boundary. These potentially unstable systems affect the relative orientation of triple orbits. In Table 2 additional columns demonstrate the shift of the mean angles, if only stable or unstable systems are selected according to the above criterium. In general, stable orbits tend to exhibit a stronger correlation as compared to the unstable ones (except for the high $\beta$ models). Retrograde orbits are more frequently found at the stability boundary, thus having a stabilizing effect as already noted by Harrington (1977). The same trend is found in the MSC data, where the mean $\langle\Phi\rangle_{\mathrm{s}}=77^{\circ}$ and $\langle\Phi\rangle_{\mathrm{u}}=84^{\circ}$, but with low statistical significance.

We argue that a considerable fraction of the higheccentricity orbits in the simulated sample are probably not long-term stable and will decay by alternative processes. Some of them might evolve into stable systems with short-period inner binaries if tidal friction will circularize and shrink orbits as discussed in Kiseleva et al. (1998). This process is especially efficient where the initial orbit inclinations are high, a condition that is often met in remnants of dynamical decaying clusters (see above).

\section{Conclusions}

We have analyzed the statistics of the angle $\Phi$ between the angular momentum vectors of inner and outer orbits in triple systems produced in direct dynamical decay simulations of few-body clusters having $3 \leq N \leq 10$. For $N=4$ and equal masses, we find that only extreme geometrical and virial initial conditions will result in significant deviations from the uncorrelated orbital momenta. With a more realistic "two-step" mass spectrum, the correlation is sensitive to the geometry and rotation of the progenitor cluster, and the $\Phi$ statistics is a powerful probe of initial cluster properties. We find that models involving a moderate oblateness of 10:1 and relative rotational energy $\beta=0.1$ result in a mean $\langle\Phi\rangle=63^{\circ} \pm 5^{\circ}$. We compare the simulated cumulative distribution function of $\Phi$ with the one constructed for 22 real multiple systems with both inner and outer visual orbits known and find a good agreement for $\Phi \leq 90^{\circ}$. An alternative sample of 135 visual triple systems with known apparent sense of revolution has $\langle\Phi\rangle=67^{\circ} \pm 9^{\circ}$, also matching the results of simulations. A decrease of the sign correlation with increasing period ratios can be noticed in the MSC data set. Also this trend can be reproduced by the dynamical 


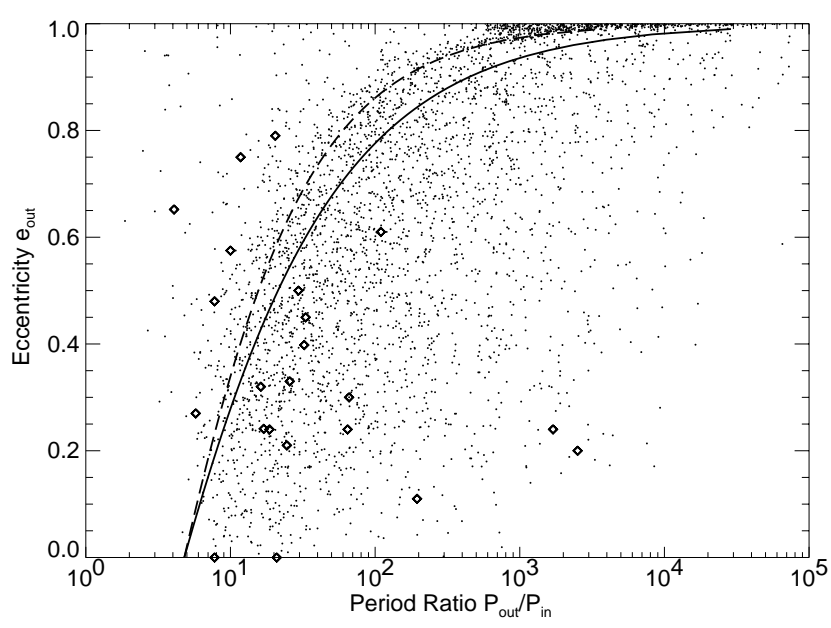

Fig. 6. Eccentricities of the outer orbit $\left(e_{\text {out }}\right)$ versus period ratios $P_{\text {out }} / P_{\text {in }}$. Dots are from all decay simulations explained in the previous section. Symbols indicate the 22 data points from MSC. The full line indicates the stability limit (6), dashed line - modified limit with $s=0.9$.

decay model simulations. However, when marginally stable triples are excluded, the mean correlation of orbital momenta increases because a large fraction of systems at the limit of stability must have more stable retrograde orbits.

It will be an important observational challenge to increase the statistics of systems with known angle $\Phi$. Fortunately, interferometers with long baselines are now coming into operation. They will resolve close sub-systems and will also increase the astrometric precision for the known visual binaries, permitting to derive astrometric orbits for the inner spectroscopic sub-systems and thus to determine $\Phi$. Coupling interferometric techniques with spectroscopy, it will be possible to resolve the ambiguity of the ascending node and thus to construct the real, "undiluted" distribution of $\Phi$. This, in turn, will enable us to put strong constrains on the properties of progenitor clusters, as well as on secular evolutionary processes in triples and multiples.

Acknowledgements. N. Shatsky has contributed to updating of the MSC database and has kindly provided his data on the relative motion in wide visual multiples, partially used here. Thanks to R. Mardling and S. Aarseth for their comments on the triple stability analysis.

\section{References}

Anosova, J. P. 1989, Comments Astroph., 14, 17

Batten, A. H. 1973, Binary and Multiple Star Systems (Pergamon Press, Oxford)

Bodenheimer, P. 1978, ApJ, 224, 988

Bonnell, I., \& Bate, M. R. 1994, MNRAS, 269, L45

Durisen, R. H., Sterzik, M. F., \& Pickett, B. K. 2001, A\&A, 371,952

Fekel, F. C. 1981, ApJ, 246, 879

Harrington, R. S. 1977, AJ, 82, 753

Hartkopf, W. I., \& Mason, B. D. 2001, AJ, in press (http://ad.usno.navy.mil/ad/wds/orb6.html)

Kiseleva, L. G., Eggelton, P. P., \& Mikkola, S. 1998, MNRAS, 300,292

Kroupa, P., \& Burkert, A. 2001, ApJ, 555, 945

Mardling, R. A. 2001, in Evolution of Binary and Multiple Star Systems, ed. S. Rappaport et al., ASP Conf. Ser., 229

Mardling, R. A., \& Aarseth, S. J. 2001, MNRAS, 321, 398

Mazeh, T., Latham, D. W., Goldberg, E., et al. 2001, MNRAS, 325,343

Mikkola, S., \& Aarseth, S. J. 1993, Cel. Mech. Dyn. Astr., 57, 439

Shatsky, N. 2001, A\&A, 380, 238

Sterzik, M. F., \& Durisen, R. H. 1995, A\&A, 304, L9

Sterzik, M. F., \& Durisen, R. H. 1998, A\&A, 339, 95

Tokovinin, A. A. 1993, Astron. Lett., 19, 383

Tokovinin, A. A. 1997, A\&AS, 124, 75

Tokovinin, A. A., \& Smekhov, M. G. 2002, A\&A, 382, 118

Worley, C. 1967, in On the evolution of double stars, ed. J. Dommanget, Comm. Roy. Obs. Belg., Ser. B, 17, 221 